\title{
Brain afferents to the medullary dorsal reticular nucleus: a retrograde and anterograde tracing study in the rat
}

\author{
Armando Almeida, ${ }^{1,2, *}$ Angeles Cobos, ${ }^{3}$ Isaura Tavares ${ }^{1}$ and Deolinda Lima ${ }^{1}$ \\ ${ }^{1}$ Institute of Histology and Embryology, Faculty of Medicine, Porto, Portugal \\ ${ }^{2}$ Life and Health Sciences Research Institute, School of Health Sciences, University of Minho, Braga, Portugal \\ ${ }^{3}$ Department of Morphological Sciences, Faculty of Medicine and Odontology, University of Santiago de Compostela, Spain
}

Keywords: amygdala, antinociceptive system, extrapyramidal system, hypothalamus, pain modulation, prefrontal cortex, sensorimotor cortex

\begin{abstract}
The medullary dorsal reticular nucleus (DRt) was recently shown to belong to the supraspinal pain control system; neurons within this nucleus give origin to a descending projection that increases spinal nociceptive transmission and facilitates pain perception [Almeida et al. (1999), Eur. J. Neurosci., 11, 110-122]. In the present study, the areas of the brain that may modulate the activity of DRt neurons were investigated by using of tract-tracing techniques. Injection of a retrograde tracer into the DRt resulted in labelling in multiple areas of the brain. In the contralateral orbital, prelimbic, infralimbic, insular, motor and somatosensory cortices labelling was prominent, but a smaller ipsilateral projection from these same areas was also detected. Strong labelling was also noted in the central amygdaloid nucleus, bed nucleus of stria terminalis and substantia innominata. Labelled diencephalic areas were mainly confined to the hypothalamus, namely its lateral and posterior areas as well as the paraventricular nucleus. In the mesencephalon, the periaqueductal grey, red nucleus and deep mesencephalic nucleus were strongly labelled, whereas, in the brainstem, the parabrachial nuclei, rostroventromedial medulla, nucleus tractus solitarius, spinal trigeminal nucleus, and the parvocellular, dorsal, lateral and ventral reticular nuclei were the most densely labelled regions. All deep cerebellar nuclei were labelled bilaterally. These data suggest that the DRt integrates information from the somatosensory, antinociceptive, autonomic, limbic, pyramidal and extrapyramidal systems while triggering its descending facilitating action upon the spinal nociceptive transmission.
\end{abstract}

\section{Introduction}

The medullary dorsal reticular nucleus (DRt; Lima, 1990; Swanson, 1998 ) is an area strongly involved in nociceptive processing. Anatomical studies have revealed a closed reciprocal circuit between the DRt and spinal dorsal horn. Descending DRt fibres make asymmetrical, presumably excitatory (Shepherd, 1990; Todd \& Spike, 1993) synaptic contacts upon lamina I spinal cells that, in turn, project back to the nucleus (Almeida et al., 1993); in addition, ascending fibres originating in laminae I and IV-V neurons make asymmetrical synaptic contacts upon DRt neurons projecting back to the same spinal laminae (Almeida et al., 2000). These data revealed the existence of a putative reverberative excitatory loop between the DRt and spinal lamina I. The ascending spinal projection targeting the DRt conveys nociceptive information as a large percentage of lamina I neurons targeting the DRt (Almeida \& Lima, 1997) and most neurons recorded in the DRt (Villanueva et al., 1988, 1989; Roy et al., 1992) are activated by noxious stimulation. Regarding the descending action of the loop, different lines of evidence have shown that the DRt has a facilitating action upon pain perception: (i) glutamate stimulation of the DRt resulted in a decrease of the tail-flick latency

Correspondence: Professor Armando Almeida, at *present address below. E-mail: aalmeida@ecsaude.uminho.pt

* Present address: Escola de Ciências da Saúde, CP II-Piso 3, Universidade do Minho, Campus de Gualtar, 4710-057 Braga, Portugal

Received 1 March 2002, revised 15 April 2002, accepted 7 May 2002
(Almeida et al., 1996); (ii) electrical or chemical lesioning of the nucleus increased the tail-flick latency and the response temperature in the tail-flick and hot plate tests, respectively (Almeida et al., 1996); (iii) lesioning the DRt decreased the pain-like response in both the acute and inflammatory pain phases of the formalin test (Almeida et al., 1999a); (iv) the attenuation in nociceptive behaviour resulting from DRt lesioning is accompanied by a decrease in noxious-evoked c-fos spinal expression in laminae I-II and IV-V (Almeida et al., 1999a); (v) glutamate stimulation of the DRt resulted in the facilitation (increase) of the postdischarge of wide-dynamic-range (WDR) spinal nociceptive neurons (Dugast et al., 2000), an effect that is transiently reversed by DRt administration of the local analgesic lidocaine (C.Dugast, A.Almeida \& D.Lima, unpublished data); (vi) DRt glutamate-activation reduced the stimulation frequency and/or the number of stimulation pulses needed to trigger wind-up in WDR neurons (Dugast et al., 2001). The above data indicate that the nucleus has a pro-nociceptive action that is mediated by a descending excitatory projection impinging upon spinal nociceptive neurons (reviewed by Lima \& Almeida, 2002).

A large number of studies have been dedicated to the descending nociceptive inhibitory influences triggered by the periaqueductal grey matter (PAG) -rostroventromedial medulla (RVM) -spinal cord circuit and by the noradrenergic pontomesencephalic nuclei projecting to the cord (for extensive reviews see Basbaum \& Fields, 1984; Hammond, 1986; Gebhart \& Randich, 1990; Jones, 1992). In contrast, the descending facilitating influences, whose existence 
82 A. Almeida et al.

TABLE 1. Density of retrogradely labelled neurons after small (rat 691) and large (rat 536) CTb injections restricted to the Drt

\begin{tabular}{|c|c|c|c|c|}
\hline \multirow{2}{*}{$\begin{array}{l}\text { Location of } \\
\text { labelled neurons }\end{array}$} & \multicolumn{2}{|c|}{ Small injection of $\mathrm{CTb}$} & \multicolumn{2}{|c|}{ Large injection of $\mathrm{CTb}$} \\
\hline & Ipsilateral & Contralateral & Ipsilateral & Contralateral \\
\hline \multicolumn{5}{|l|}{ Telencephalon } \\
\hline Orbital cortex & & ++ & & ++ \\
\hline Prelimbic and infralimbic cortices & & ++ & & ++++ \\
\hline Secondary motor cortex & + & +++ & + & +++ \\
\hline Primary motor cortex & + & ++++ & ++ & +++++ \\
\hline Secondary somatosensory cortex & + & ++++ & + & ++++ \\
\hline Primary somatosensory cortex & + & ++++ & + & ++++ \\
\hline Dysgranular/granular insular cortices & + & ++++ & +++ & +++++ \\
\hline Agranular insular cortex & +++ & ++++ & +++ & +++++ \\
\hline Cingulate cortex & & & & + \\
\hline Retrosplenial granular cortex & & + & & + \\
\hline Secondary auditory cortex & & & & ++++ \\
\hline Primary auditory cortex & & & & ++ \\
\hline Perirhinal cortex & & + & + & +++ \\
\hline Ectorhinal cortex & & + & & ++ \\
\hline Parietal association cortex & & & & + \\
\hline Temporal association cortex & & + & & ++ \\
\hline Lateral $/$ medial preoptic area & & & + & \\
\hline Bed nucleus stria terminalis & + & & ++++ & + \\
\hline Substantia innominata & ++ & & ++ & \\
\hline Globus pallidus, lateral & ++ & & ++ & \\
\hline Central amygdaloid nucleus & +++++ & + & +++++ & + \\
\hline \multicolumn{5}{|l|}{ Diencephalon } \\
\hline Paraventricular hypothalamic nucleus & +++ & ++ & ++++ & +++ \\
\hline Parasubthalamic nucleus & + & & +++ & +++ \\
\hline Zona incerta & ++ & + & ++ & + \\
\hline Posterior hypothalamic area & & & ++ & ++ \\
\hline Lateral hypothalamic area & ++++ & ++ & ++++ & +++ \\
\hline Parafascicular thalamic nucleus & + & + & + & \\
\hline \multicolumn{5}{|l|}{ Mesencephalon } \\
\hline Red nucleus & & ++++ & & ++++ \\
\hline Substantia nigra, compact & + & & ++ & + \\
\hline Substantia nigra, reticular & ++ & & ++ & \\
\hline Retrorubral field & & & ++ & + \\
\hline Subpeduncular tegmental nucleus & & & + & + \\
\hline Deep mesencephalic nucleus & +++ & +++ & ++++ & +++ \\
\hline Dorsal raphe nucleus & ++ & ++ & & \\
\hline Periaqueductal grey, dorsomedial & + & +++ & & \\
\hline Periaqueductal grey, lateral & ++ & ++ & ++++ & ++++ \\
\hline Periaqueductal grey, ventrolateral & +++ & + & ++++ & ++ \\
\hline Cuneiform nucleus & + & & ++ & + \\
\hline \multicolumn{5}{|l|}{ Pons } \\
\hline Reticulotegmental nucleus of the pons & ++ & + & + & \\
\hline Basilar pontine nuclei & ++++ & +++++ & & \\
\hline Pontine reticular nucleus, oral & & + & ++ & + \\
\hline Principal sensory trigeminal nucleus & ++ & + & & \\
\hline Subcoeruleus nucleus, ventral & +++ & & +++ & + \\
\hline Mesencephalic trigeminal nucleus & + & & ++ & + \\
\hline Motor trigeminal nucleus & + & & & \\
\hline Pontine reticular nucleus, caudal & ++ & ++ & ++ & ++ \\
\hline Locus coeruleus & + & + & + & + \\
\hline Kölliker-Fuse nucleus & ++ & ++ & +++ & ++ \\
\hline Parabrachial nuclei & +++ & ++ & ++++ & +++ \\
\hline A5 noradrenergic cell group & +++ & + & +++ & ++ \\
\hline \multicolumn{5}{|l|}{ Cerebellum } \\
\hline Interposed cerebellar nucleus & ++++ & & ++++ & + \\
\hline Lateral (dentate) cerebellar nucleus & ++ & & ++ & \\
\hline Medial (fastigial) cerebellar nucleus & ++ & ++ & + & ++++ \\
\hline \multicolumn{5}{|l|}{ Medulla oblongata } \\
\hline Rostroventrolateral reticular nucleus & + & + & + & + \\
\hline Raphe magnus nucleus & ++ & ++++ & & \\
\hline Raphe pallidus nucleus & ++ & ++ & & \\
\hline Raphe obscurus nucleus & + & ++ & & \\
\hline Vestibular nuclei & + & + & + & + \\
\hline Dorsal paragigantocellular nucleus & + & + & + & + \\
\hline Lateral paragigantocellular nucleus & ++ & ++ & ++++ & ++++ \\
\hline Gigantocellular reticular nucleus, alpha & ++ & + & ++++ & ++++ \\
\hline
\end{tabular}


TABLE 1. (continued)

\begin{tabular}{|c|c|c|c|c|}
\hline \multirow{2}{*}{$\begin{array}{l}\text { Location of } \\
\text { labelled neurons }\end{array}$} & \multicolumn{2}{|c|}{ Small injection of $\mathrm{CTb}$} & \multicolumn{2}{|c|}{ Large injection of $\mathrm{CTb}$} \\
\hline & Ipsilateral & Contralateral & Ipsilateral & Contralateral \\
\hline Gigantocellular reticular nucleus, ventral & ++ & + & ++ & ++ \\
\hline Gigantocellular reticular nucleus & ++ & ++ & +++ & ++ \\
\hline Intermediate reticular nucleus & ++ & + & +++ & ++ \\
\hline Dorsal reticular nucleus & +++ & +++ & ++++ & ++++ \\
\hline Area postrema & + & ++ & & \\
\hline Nucleus of Roller & & & + & ++ \\
\hline Hypoglossal nucleus & + & + & + & + \\
\hline Dorsal motor nucleus of vagus & + & + & + & + \\
\hline Nucleus of the solitary tract, ventrolateral & ++++ & ++++ & +++++ & +++++ \\
\hline Nucleus of the solitary tract & ++++ & ++++ & +++++ & ++++ \\
\hline Nucleus cuneatus & ++ & & +++ & \\
\hline Paratrigeminal nucleus & ++++ & & ++++ & + \\
\hline Spinal trigeminal nucleus & +++++ & ++ & +++++ & ++ \\
\hline Ventral reticular nucleus & + & ++ & ++ & ++ \\
\hline Caudal ventrolat. ret. form., lateral & + & & +++ & ++ \\
\hline A1 noradrenergic cell group & ++ & & ++ & ++ \\
\hline Lateral reticular nucleus & ++ & + & ++ & + \\
\hline Inferior olive & & +++++ & & \\
\hline Parvocellular reticular nucleus & +++++ & +++ & +++++ & ++++ \\
\hline
\end{tabular}

Intensity of label: +++++, very dense; ++++, dense; +++, numerous; ++, few; + rare.
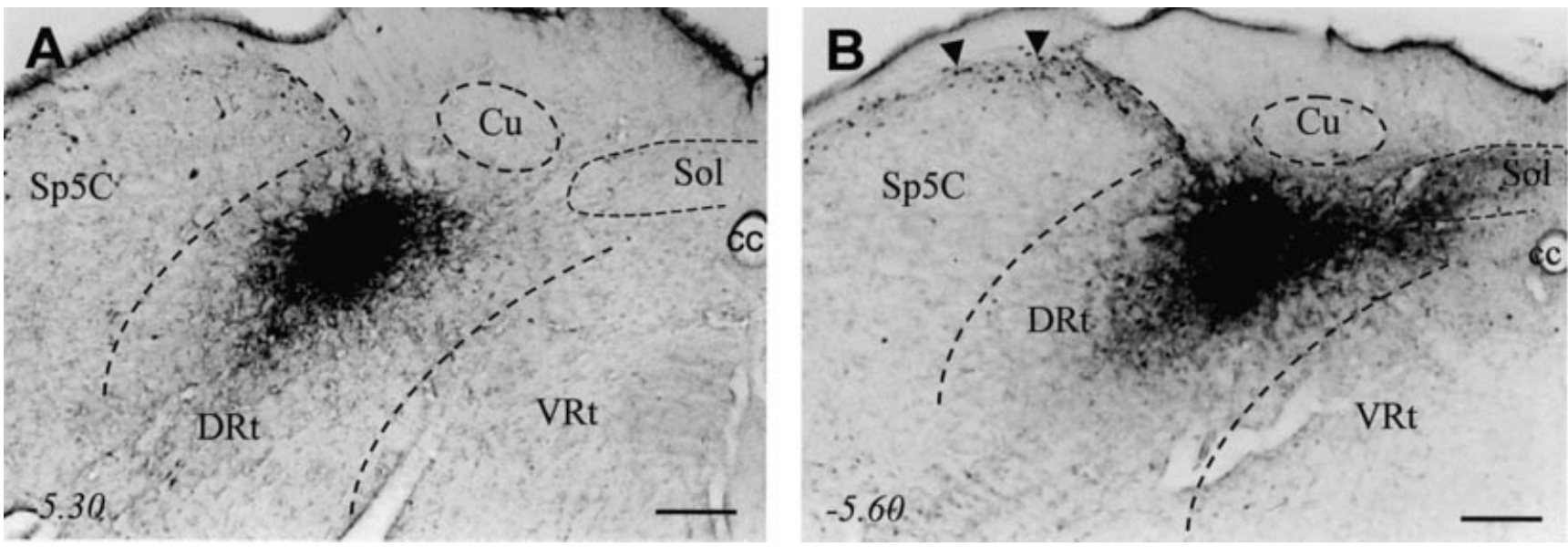

FIG. 1. Photomicrographs, each showing the coronal section presenting the largest tracer spread resulting from DRt injection with the retrograde tracer Ctb: (A) in a typical small rat (rat 691) and (B) in a typical large rat (rat 536). In both injections, CTb extends through the DRt but is restricted to the limits of

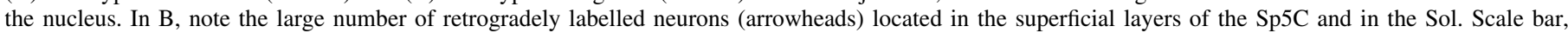
$150 \mu \mathrm{m}$. For abbreviations, see list.

were occasionally reported in the eighties, was only recently subjected to detailed research (reviews by Fields \& Basbaum, 1999; McNally, 1999; Millan, 1999; Urban \& Gebhart, 1999; Lima \& Almeida, 2002). Both inhibitory and facilitating descending actions were shown to be: (i) increased in the same nociceptive situation (e.g. chronic/neuropathic pain; Cervero et al., 1991; Schaible et al., 1991; Bian et al., 1998; Kauppila et al., 1998); (ii) triggered from the same brain areas (RVM, Fields et al., 1983; Zhuo \& Gebhart, 1992, 1997; Wei et al., 1999; nucleus tractus solitarius, Ren et al., 1990; DRt, Bouhassira et al., 1992; Almeida et al., 1996, 1999a); and (iii) triggered from the same type of descending projection (e.g. noradrenergic descending pathways; Proudfit \& Hammond, 1981; Yaksh, 1985; Fasmer et al., 1986; Tjølsen et al., 1991; Martin et al., 1999). Thus, it is a complex balance of facilitating and inhibiting descending influences upon the nociceptive transmission at the spinal dorsal horn level that determines pain modulation, the final action being dependent on the specific characterization of each painful condition (Lima, 1998; Fields \& Basbaum, 1999; Lima \& Almeida, 2002).

In order to ascertain the pattern of anatomical connections that may modulate the activity of the nociceptive-facilitating neurons present in the DRt, an extensive study of the brain areas that project to the nucleus was carried out. Some of the data have been presented previously in abstract form (Almeida et al., 1999b).

\section{Materials and methods}

The experiments followed the regulations of local authorities for handling laboratory animals, the European Community Council Directive 86/609/EEC and the ethical guidelines for the study of experimental pain in conscious animals (Zimmermann, 1983). 

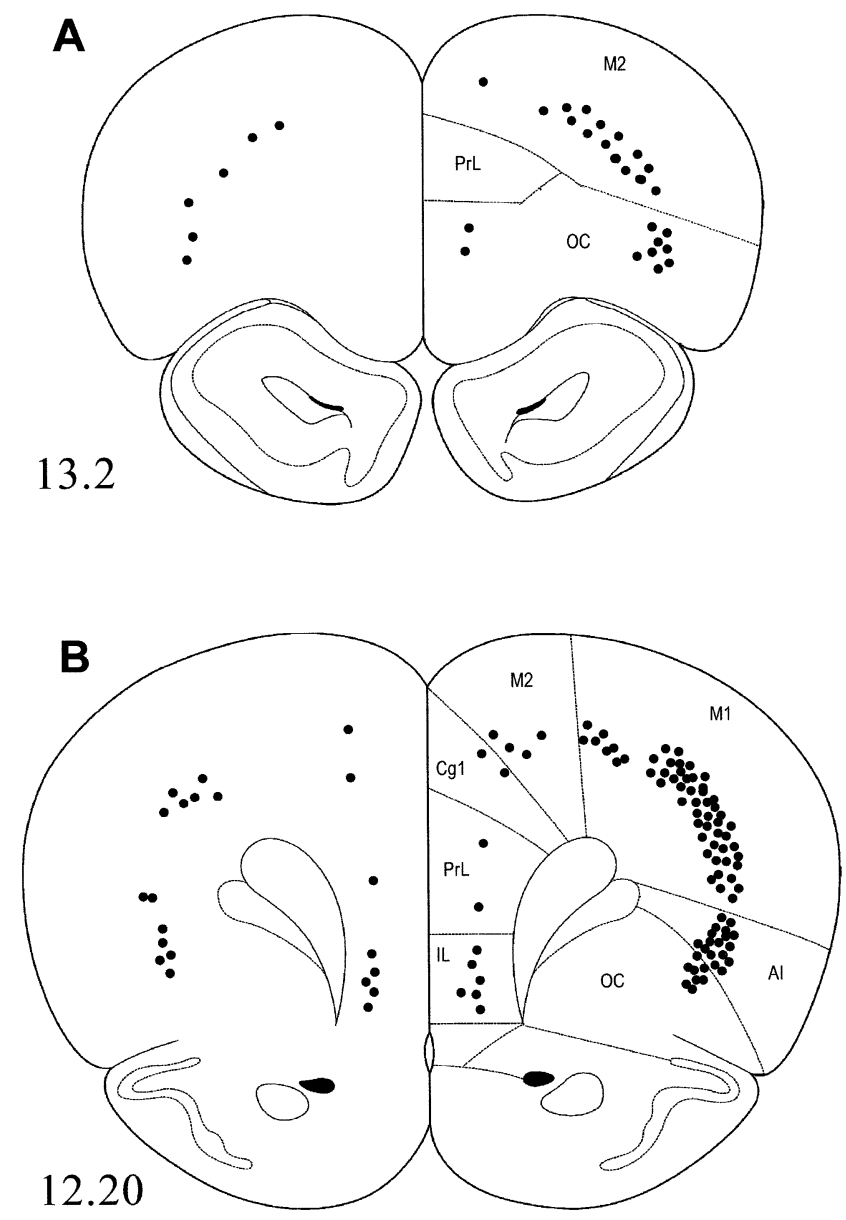

FIG. 2A and B. Locations of retrogradely labelled cells plotted on representative coronal sections through the brain of rat 536. The injection site is shown photographically in Fig. 1B. Each dot $(-)$ and triangle $(\boldsymbol{\Lambda})$ represents 1 and 5 neurons, respectively. (A and B) Sections at 13.2 and $12.20 \mathrm{~mm}$ rostral to the interaural line. For abbreviations, see list. freezing microtome at $50 \mu \mathrm{m}$. One in every three brain sections was immunoreacted for $\mathrm{CTb}$ and counterstained using the formol-thionin technique (Donovick, 1974) and one in every three sections was only immunoreacted for $\mathrm{CTb}$. $\mathrm{CTb}$ was revealed using the immunoperoxidase technique, as previously described (Almeida \& Lima, 1997; Almeida et al., 2000). Briefly, sections were first incubated overnight at room temperature in a goat primary polyclonal antibody against $\mathrm{CTb}$ (List Biological Products, USA) at $1: 40000$ in $0.1 \mathrm{M}$ phosphate buffer saline containing $0.3 \%$ Triton X-100 (PBST). After washing repeatedly in PBST, the sections were incubated for $1 \mathrm{~h}$ in PBST containing a biotinylated antigoat antibody raised in horse (1:200; Vector Laboratories, Burlingame, USA). Sections were then washed again and incubated for $1 \mathrm{~h}$ in PBST containing the avidin-biotin complex ( $1: 200$, Vector Laboratories, USA). After washing in $0.1 \mathrm{M}$ tris- $\mathrm{HCl}$, pH 8.2, peroxidase was revealed using $0.0125 \% 3,3^{\prime}$ diaminobenzidine tetrahydrochloride (DAB; Sigma Immunochemicals, St Louis, USA) and $0.025 \% \mathrm{H}_{2} \mathrm{O}_{2}$ in the same buffer. The sections were then dehydrated and mounted in Eukitt. Cytoarchitectonic diagrams of the injection sites were made with the aid of a camera lucida. Retrogradely labelled neurons in brain areas projecting to the DRt were located and superimposed on projection drawings of selected representative sections from the atlas of Paxinos \& Watson (1998).

\section{Anterograde-tracing control studies}

Injection of the anterograde tracer biotinylated-dextran amine (BDA) was made in several DRt-projecting brain nuclei, in order to establish their termination areas in the nucleus. The paraventricular hypothalamic nucleus $(\mathrm{Pa})$, the lateral hypothalamic area $(\mathrm{LH})$, the ventrolateral periaqueductal grey matter (VLPAG), the red nucleus (RD) and the lateral cerebellar nucleus of male Wistar rats were injected iontophoretically in the left side of the brain, under halothane anaesthesia (see above), with a $10 \%$ PB buffer, pH 7.2, using a positive DC current of $2.5 \mu \mathrm{A}$ for $20-30 \mathrm{~min}$. Ten days later the rats were anaesthetized and perfused as described above. Serial $50 \mu \mathrm{m}$ thick frozen coronal sections of the injection site and caudal medulla were immunoreacted using the avidin-biotin complex method and stained with DAB (see Almeida et al., 1995), the former sections being further counterstained with formol-thionin. Fibres and terminal arborizations labelled anterogradely in the DRt were plotted using camera lucida drawings.

\section{Results}

\section{Injection sites}

$\mathrm{CTb}$ injection sites showed a compact dark zone surrounded by a narrow rim without neuronal labelling, in which dark zones intermingled with lighter zones (Fig. 1). More peripherally, a few perikarya were labelled as a consequence of tracer uptake from the central area. The injection site was considered to encompass only the central dark area and surrounding rim. The injections were all placed inside the limits of the DRt and thus were all considered for analysis.

BDA injection sites also showed the compact dark central zone surrounded by a narrow rim of scarcely labelled neurons. In accordance with previous technical studies, only the central region and the rim were included in the injection site (Veenman et al., 1992; Wouterlood \& Jorritsma-Byham, 1993). In the animals analysed, injections were placed inside the anatomical borders of the left $\mathrm{Pa}$, LH, RD and VLPAG. immersed in the fixative for $4 \mathrm{~h}$, then in $30 \%$ sucrose in PB at $4{ }^{\circ} \mathrm{C}$ for 2-3 days. Coronal sections of the entire brain were cut on a 

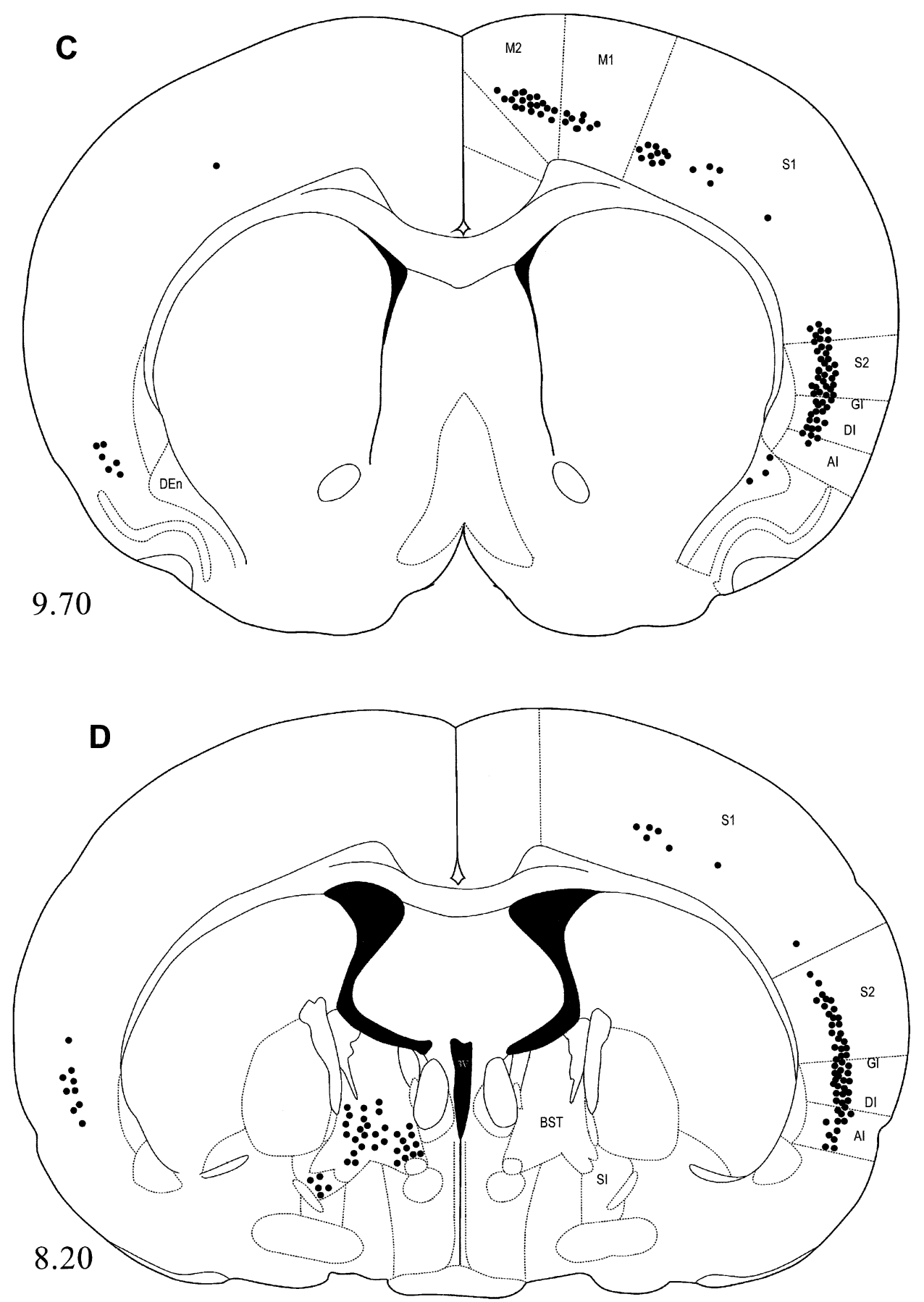

FIG. 2C and D. (Fig. 2 continued) Sections at 9.70 and $8.20 \mathrm{~mm}$ rostral to the interaural line. For details see legend to Fig. 2A and B, and abbreviations list.

\section{Retrograde labelling}

CTb-labelled neurons projecting to the DRt were distributed along the rostrocaudal extension of the brain, particularly in the cortex (contralateral) and brainstem, although high numbers of neurons were also found in some nuclei of the ventral telencephalon and diencephalon. A systematic analysis of the location and density of neurons projecting to the DRt is described in Table 1, whereas in Fig. 2 the precise locations of retrogradely labelled neurons are plotted on brain charts.

\section{Telencephalon}

In the prefrontal cortex, a bilateral projection to the DRt was shown to occur from the prelimbic (PrL), infralimbic (IL) (Figs 2A and B, and 3A), orbital (OC; Fig. 2A and B), and cingulate (Cg1) (Fig. 2B) cortices, although with a contralateral predominance. In the motor cortex, a higher number of neurons was present in the primary (M1) than in secondary (M2) motor cortices and, in both cases, they were predominantly located on the contralateral hemisphere (Fig. 2A-C). Regarding the somatosensory cortex, a large number of neurons were 

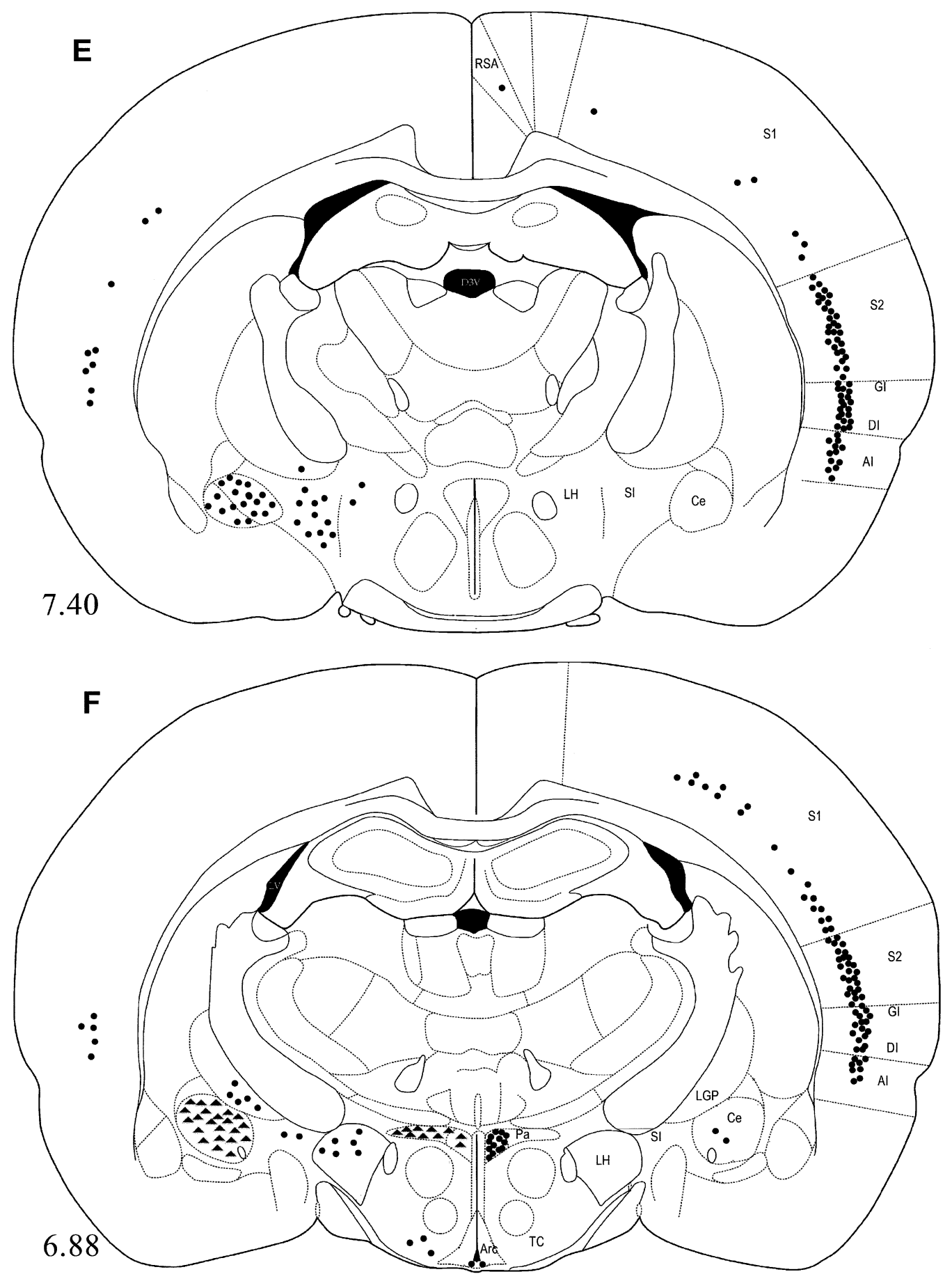

FIG. 2E and F. (Fig. 2 continued) Sections at 7.40 and $6.88 \mathrm{~mm}$ rostral to the interaural line. For details see legend to Fig. 2A and B, and abbreviations list.

present, mainly in the contralateral secondary somatosensory cortex (S2) and, in smaller numbers, in the contralateral primary somatosensory cortex (S1) (Fig. 2C-F), barrel field and facial regions. A small ipsilateral projection from the $S_{1}$ and $S_{2}$ cortical areas was also present (Fig. 2E). The forelimb, hindlimb and thorax regions showed very few or no cells. In the insular cortices, labelled neurons were present in all sections. Although the majority were located contralaterally ( Fig. 2B-F), an ipsilateral projection was also consistently observed (Figs 2B-F and 3B).

In subcortical telencephalic areas, an impressive number of labelled neurons was located in the ipsilateral bed nucleus of the stria terminalis (BST; Fig. 2D). In the amygdala, a very strong projection was shown to occur, almost exclusively, along the entire rostrocaudal extent of the central amygdaloid nucleus (Ce), 


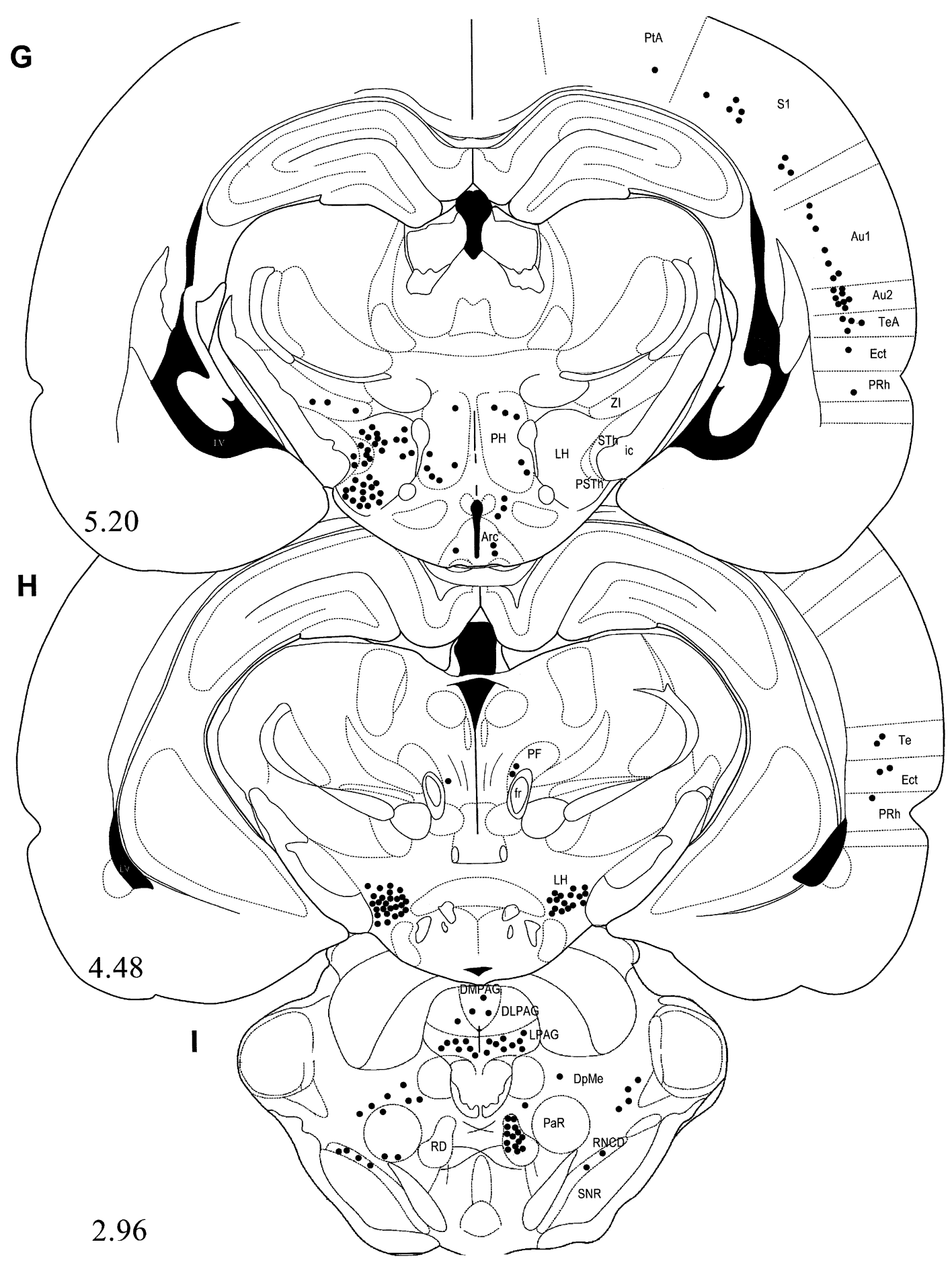

FIG. 2G-I. (Fig. 2 continued) Sections at 5.20, 4.48 and $2.96 \mathrm{~mm}$ rostral to the interaural line. For details see legend to Fig. 2A and B, and abbreviations list.

ipsilaterally (Figs $2 \mathrm{E}$ and $\mathrm{F}$, and $3 \mathrm{C}$ ). Numerous neurons projecting to the DRt were also noted in the ipsilateral substantia innominata (SI; Fig. 2D-F).

\section{Diencephalon}

In the thalamus, only the parafascicular nucleus (PF) presented some labelled neurons both medially and laterally to the fasciculus retroflexus (Fig. 2H). A small projection from the zona incerta (ZI) was present ipsilaterally (Fig. 2G). On the other hand, the hypothalamus showed a large number of labelled cells located bilaterally in different subnuclei of the $\mathrm{Pa}$ (Figs $2 \mathrm{~F}$ and 3D). A great number of cells projecting to the DRt were also located in the medial and caudal portions of the LH, bilaterally (Fig. 2E-H). In the caudal hypothalamus, the posterior hypothalamic area $(\mathrm{PH})$ showed a 

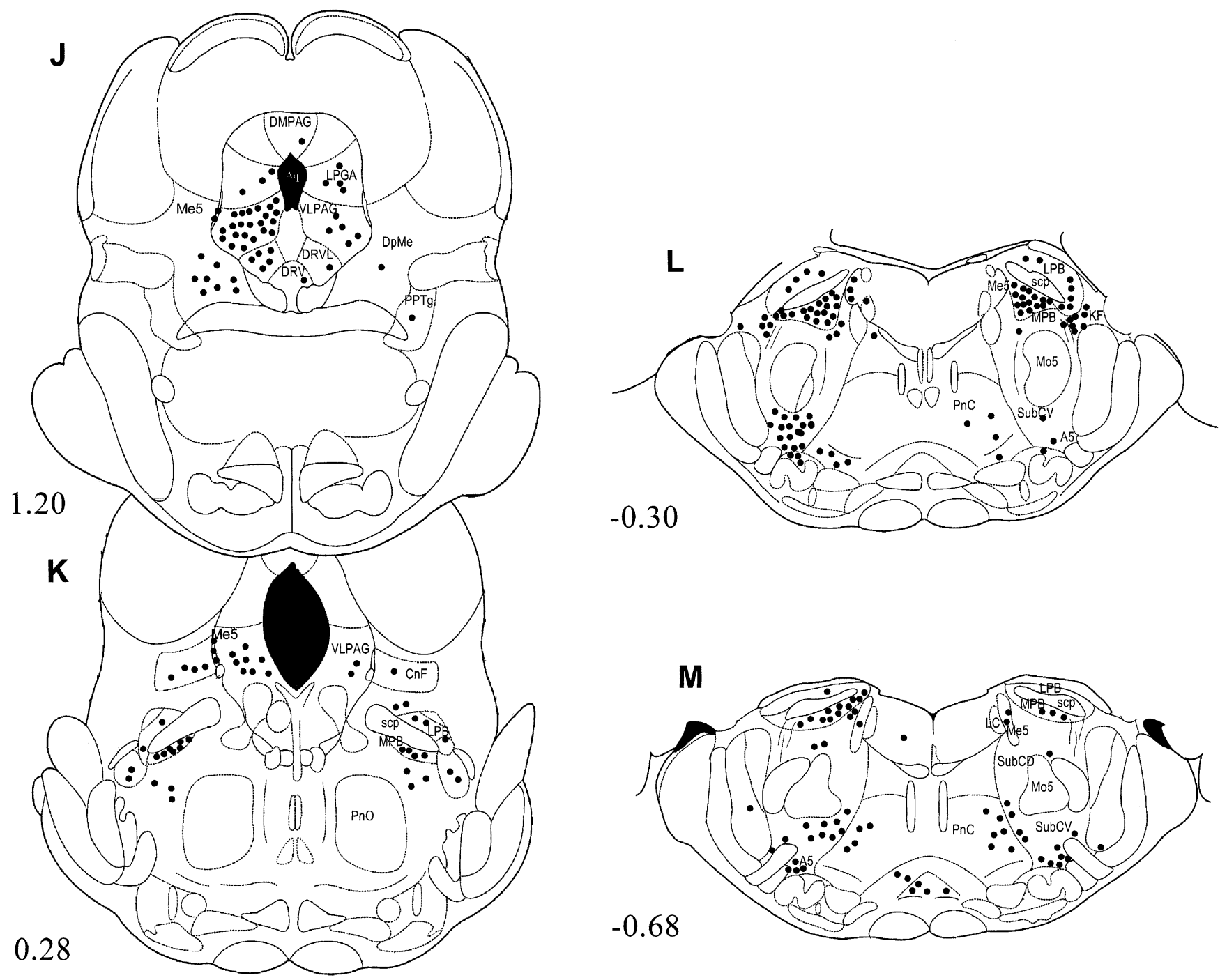

FIG. 2J-M. (Fig. 2 continued) Sections at 1.20 and $0.28 \mathrm{~mm}$ rostral, and 0.30 and $6.8 \mathrm{~mm}$ caudal to the interaural line. For details see legend to Fig. 2A and B, and abbreviations list.

moderate number of retrogradely labelled neurons, bilaterally (Fig. 2G). Fewer neurons were labelled in the arcuate nucleus (Arc) and ipsilateral tuberum cinerum (TC; Figs $2 \mathrm{~F}$ and $\mathrm{G}$ ).

\section{Mesencephalon}

Labelled cells were located in the caudalmost areas of the substantia nigra, bilaterally (Fig. 2I). In the RD, most of the large neurons labelled contralaterally were concentrated in its dorsal region (Fig. 2I). Several bilaterally located labelled neurons were observed through the rostrocaudal extension of the lateral and VLPAG (Figs $2 \mathrm{I}-\mathrm{K}$ and $3 \mathrm{E}$ ). Other areas of the PAG presented a small number of labelled neurons (Figs 2I and J). Labelled cells were scattered, mainly ipsilaterally, through the large area of the brainstem reticular formation forming the deep mesencephalic nucleus (DpMe; Figs $2 \mathrm{I}$ and $\mathrm{J}$ ), and in the cuneiform nucleus (CnF; Fig. 2K). In the dorsal raphe, most neurons were present in the ventrolateral portion of the nucleus followed by the ventral part, whereas the dorsal part of the dorsal raphe was not labelled (Fig. 2J).
Pons

In one rat (number 691, small injection), the basilar pontine nuclei (BPN) were the areas of the pons presenting the most extensive distribution of bilaterally located cells, but no cells were labelled in the other rat described in Table 1 (numb 536, large injection). The $\mathrm{CnF}$ (Fig. 2K) and the caudal pontine reticular nucleus (PnC; Fig. 2L-N) showed a moderate number of labelled neurons. A highly packed set of cells projecting ipsilaterally to the DRt was present in the mesencephalic trigeminal nucleus (Me5; Fig. 2J-M). The $\mathrm{A}_{5}$ noradrenergic group (A5), locus coeruleus (LC) and nucleus subcoeruleus (SubCV) were noradrenergic brainstem areas that presented a moderate number of labelled cells located mainly ipsilaterally (Fig. 2L-N). A large number of labelled neurons was distributed bilaterally through the subnuclei of the parabrachial nuclei (PBN), particularly in the medial PBN (MPB) and ventral part of the lateral PBN (LPB; Fig. 2K-M). Labelled neurons in the KöllikerFuse nucleus $(\mathrm{KF})$ were present bilaterally in all the appropriate sections analysed (Figs 2K and L). 

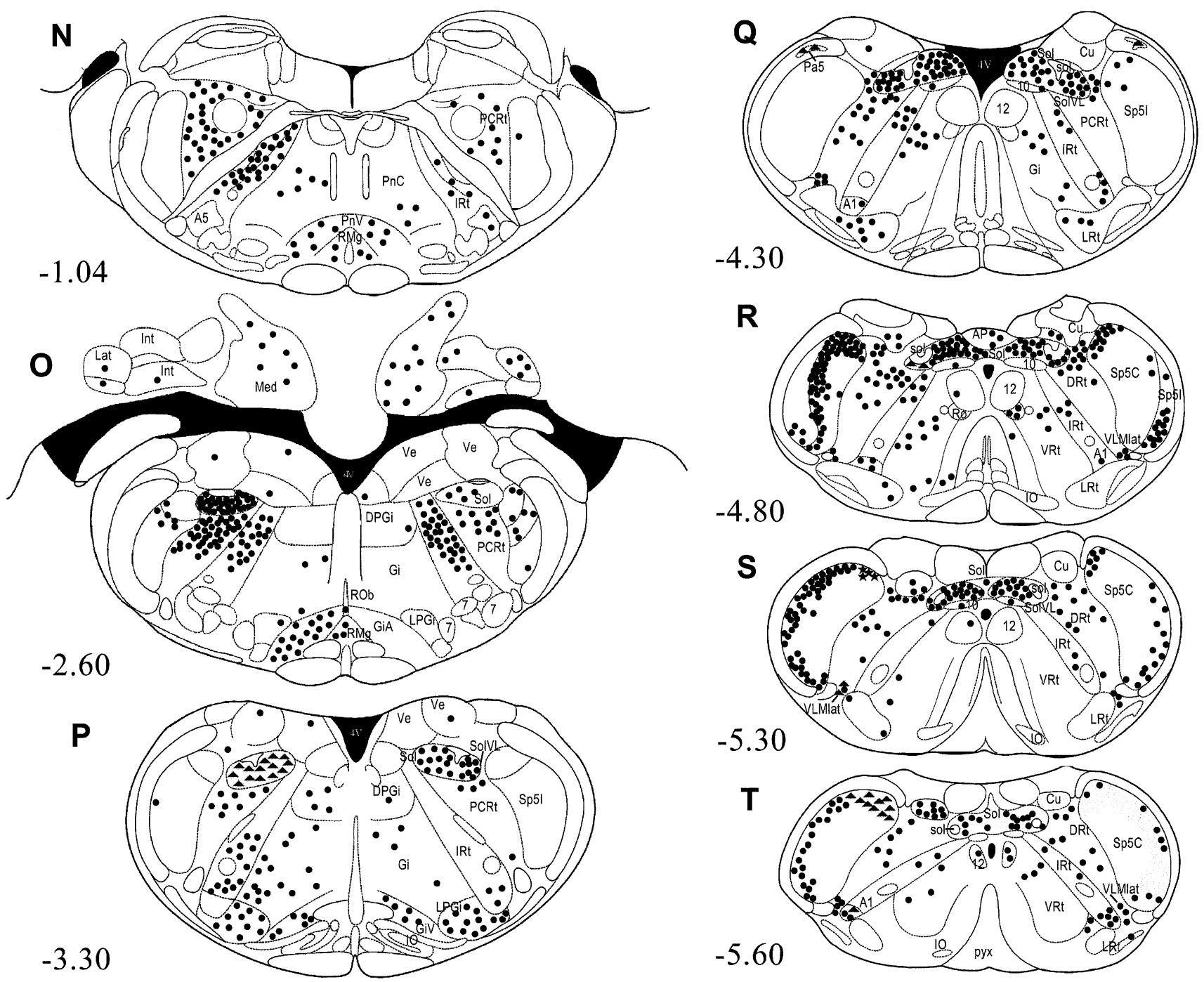

Fig. 2N-T. (Fig. 2 continued) Sections at 1.04 to $5.60 \mathrm{~mm}$ caudal to the interaural line. For details see legend to Fig. 2A and B, and abbreviations list. As before, each dot and triangle represents 1 and 5 neurons respectively. The stars in $\mathrm{S}$ each represent 10 neurons.

\section{Cerebellum}

All three major deep nuclei of the cerebellum presented a considerable number of bilaterally labelled neurons, particularly in the medial deep cerebellar nucleus (Fig. 2O).

\section{Medulla oblongata}

The gigantocellular ( $\mathrm{Gi}, \mathrm{GiA}, \mathrm{GiV})$, paragigantocellular (LPGi), lateral (LRt) and ventral (VRt) reticular nuclei presented a moderate number of labelled neurons, bilaterally (Fig. 2O-T). Although without consistent neuronal labelling in all sections analysed, all ponto-medullary raphe subdivisions contained neurons projecting to the DRt, with special emphasis on the nucleus raphe magnus (RMg; Fig. 2M-O). High numbers of neurons were located in both medial (SolM) and ventrolateral (SolVL) areas of the nucleus tractus solitarius (Sol), along its rostrocaudal extension (Figs $1 \mathrm{~B}$ and 2O-T). Numerous neurons were also located bilaterally along the rostrocaudal pontomedullary extension of the parvocellular reticular nucleus (PCRt;
Fig. 2N-Q). In rat 691 (small injection), a high number of contralaterally located neurons was present in the medial portion of the inferior olive (IO), between interaural coordinates -3.30 and $-4.68 \mathrm{~mm}$ (Paxinos \& Watson, 1998), but no labelling in this area was observed in rat 536 (large injection). A small number of labelled neurons were located in the motor nucleus of the vagal nerve (10; Fig. 2Q-S) and hypoglossal nucleus (12; Fig. 2R-T). The lateral part of the ventrolateral medulla (VLMlat) showed a bilateral projection to the DRt (Fig. 2R-T). A large number of neurons were labelled along the ipsilateral rostrocaudal extension of the caudal portion of the spinal trigeminal nucleus (Sp5C), being mainly concentrated in the most superficial laminae (Figs $1 \mathrm{~A}$ and $\mathrm{B}$, and 2R-T). Numbers were much smaller in the interpolar part of the trigeminal nucleus (Sp5I), where the neurons were preferentially located in deeper laminae (Fig. 2O-Q). The DRt, the area injected with $\mathrm{CTb}$ and the focus of this study, presented neurons of local circuit, labelled ipsilaterally, and short-projecting neurons, labelled bilaterally (Figs 2R-T and 3F). 

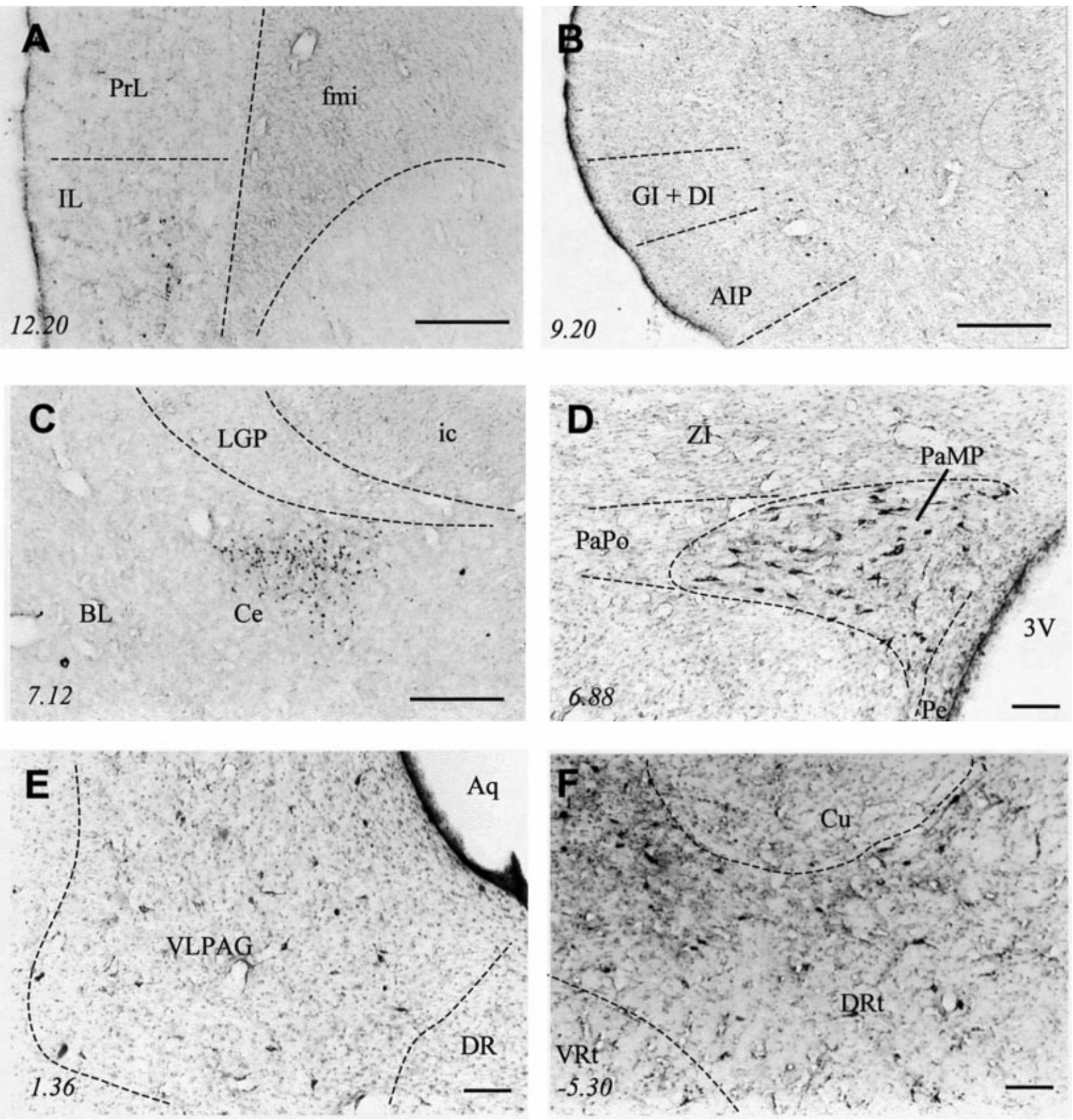

FIG. 3. Labelled neurons in the ipsilateral infralimbic (A) and insular (B) cortices, central amygdaloid (C) and paraventricular hypothalamic (D) nuclei, ventrolateral PAG (E) and contralateral DRt (F) following the large CTb injection shown in Fig. 1B. Scale bar, $150 \mu \mathrm{m}$. For abbreviations see list.

\section{Anterograde labelling}

Fibres and terminal boutons anterogradely labelled with BDA were observed mainly in the dorsal part of the DRt (DRtd) after cerebellar injections, and through the dorsoventral extension of the DRt [DRtd and ventral DRt (DRtv)] after hypothalamic (LH and Pa), VLPAG and RD (Fig. 4) injections.

Labelled fibres and terminals in the DRt occurred mainly ipsilaterally after cerebellar, hypothalamic and VLPAG injections, and exclusively contralaterally after RD injections.

\section{Discussion}

\section{Technical considerations}

Histologically, the DRt can be easily delimited from the Cu dorsally, the Sp5C laterally, the Sol dorsomedially and the VRt ventromedially. For the interpretation of the present findings, four possible sources of uncertainty inherent to the use of a tract-tracing technique were considered. First, the placement of $\mathrm{CTb}$ in the DRt; the small iontophoretic $\mathrm{CTb}$ injections resulted in restricted and perfectly defined injection sites, all located within the boundaries of the DRt 

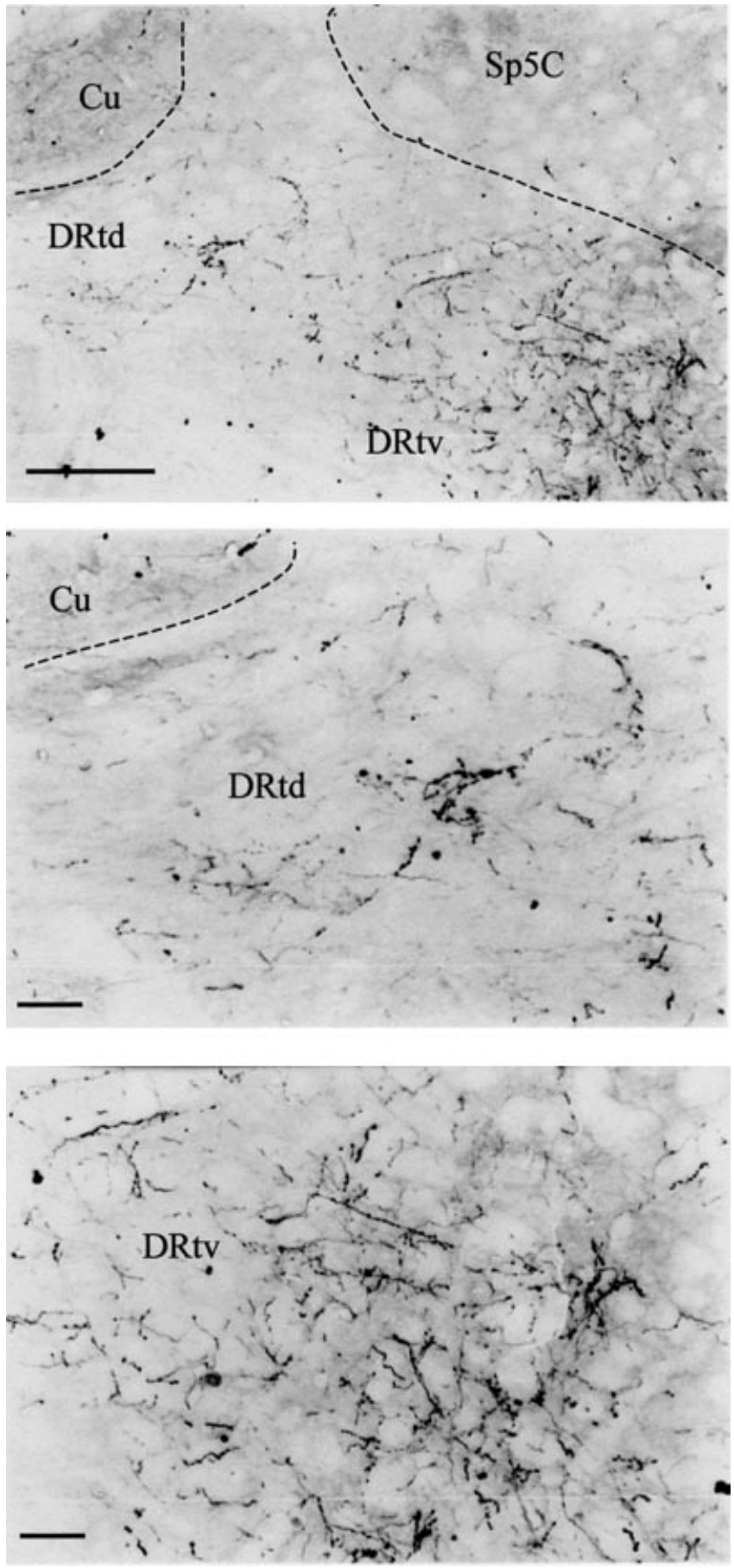

FIG. 4. Photomicrographs showing anterogradely labelled fibres and terminal boutons in the contralateral DRt following injections of BDA in the left red nucleus: (A) small magnification of the dorsoventral extension of the DRt, in which the location of the two subareas of the nucleus, the dorsal (DRtd) and the ventral (DRtv) Drt are indicated; (B and C) higher magnifications of the DRtd and DRtv areas, respectively, shown in A, which reveal details of the abundant anterograde labelling present in both areas of the nucleus. Scale bar, $150 \mu \mathrm{m}$. For abbreviations see list.

along the rostrocaudal extension of the nucleus. Larger injections, which were used in order to label larger numbers of cells, were also selected according to their inclusion inside the limits of the nucleus. Secondly, leakage of CTb: the micropipette track was examined and no leakage of CTb was detected, probably as a consequence of the small tip diameters of the micropipettes used and the time allowed for the micropipette to remain in the injection place after tracer administration. Thirdly, transneuronal transport: the possibility that trans-synaptic transport accounted for the labelling of cells receiving projections from the DRt can be ruled out, as $\mathrm{CTb}$ was shown not to be carried transneuronally after anterograde transport (Almeida et al., 1993). Fourthly, passing fibres: the possibility that fibres travelling in the injected DRt areas may contribute to some of the retrograde labelling observed does not need to be considered. In fact, previous observations have shown that no spinal labelling occurs after $\mathrm{CTb}$ injections into the spinothalamic tract (Lima et al., 1991), indicating that $\mathrm{CTb}$ is not picked up by passing fibres. Moreover, it has been demonstrated that, with $\mathrm{CTb}$, anterograde or retrograde tracing by passing fibres does not occur when iontophoretic injections are made (Luppi et al., 1990; Angelucci et al., 1996). Taking into account all the above facts, it was concluded that DRt injections resulted exclusively in $\mathrm{CTb}$ retrograde labelling in the brain after tracer uptake by afferent terminals terminating in the DRt. Therefore, it was not considered necessary to make control $\mathrm{CTb}$ injections in medullary nuclei bordering the DRt.

A surprising result was the retrograde labelling present in the BPN and IO in rat 691 (Table 1). Spread of the tracer to nuclei close to the DRt cannot account for this observation as this rat received a small and well located injection. It is possible that this finding is due to the different rostrocaudal centre of the $\mathrm{CTb}$ injection (Fig. 1, interaural coordinates: -5.30 for rat 691 vs. -5.60 for rat 536) and, consequently, reflects different anatomical loci inside the DRt.

\section{Brain afferents to the DRt: anatomical considerations}

This is the first study addressing the systematic evaluation of the brain areas projecting to the DRt, through tracer injections applied directly to the nucleus. The only other work with retrograde tracer administration to the DRt was a detailed study on the cortical projections to the nucleus (Desbois et al., 1999).

\section{Telencephalon}

To the best of our knowledge, the projections from orbital cortex, globus pallidus and substantia innominata to the DRt were reported here for the first time.

Although our data are in general in accordance with the work of Desbois et al. (1999) concerning the dense afferent projection to the DRt from widespread contralateral cortical areas like the M1, M2, S1, S2 and insular cortices, we observed further labelling in other areas of the cortex. In fact, a small but consistent projection from the ipsilateral hemisphere of those cortical areas and an important projection from the contralateral prelimbic, infralimbic and orbital cortices are also described in the present study. The variation reported in these two studies probably reflect different sensitivities of the retrograde tracers used: wheat germ agglutinin-apo horseradish peroxidase-gold (WGA-ApoHRP-Au; Desbois et al. 1999) and CTb (the present study). If this is the case, we can conclude that $\mathrm{CTb}$ is a more sensitive tracer than WGA-ApoHRP-Au, especially if we take into account that in both studies the injections were restricted to the DRt and our small injections seem to be smaller than those in the other study. Although no direct comparisons have been made between the two tracers, this hypothesis is supported by retrogradetracing studies which clearly showed that, in the spinothalamic and spino-DRt pathways, CTb labelled significantly more spinal cells than the tracers wheat germ agglutinin-horseradish peroxidase 
(WGA-HRP) or free HRP after injections of similar size in the thalamus or DRt (Lima \& Coimbra, 1988; Lima, 1990).

\section{Diencephalon}

To the best of our knowledge, the projections from the PF, posterior hypothalamic area and ZI, to the DRt were reported here for the first time. Interestingly, the PF is also one of the major efferent targets of the DRt in the brain (Villanueva et al., 1997), thereby suggesting the existence of a feedback loop between the DRt and PF.

The important projections originated in the $\mathrm{Pa}$ and $\mathrm{LH}$ have been referred to in previous anterograde tracing studies (Luiten et al., 1985; Allen \& Cechetto, 1992); however, these only reported the strong ipsilateral projection. Such variability might be ascribed again to the injection site or tracer sensitivity. Indeed, it is possible that the injections of the anterograde tracer Phaseolus vulgaris-leucoagglutinin (PHA-L) in the $\mathrm{Pa}$ did not reach the neurons originating the contralateral projection (Luiten et al., 1985). In the case of the WGAHRP injections in the $\mathrm{LH}$, it is possible that the tracer lacks sufficient sensitivity to label the contralateral side of the projection (Allen \& Cechetto, 1992).

\section{Mesencephalon}

To the best of our knowledge, the projections from the substantia nigra, RD, dorsal raphe nucleus and mesencephalic trigeminal nucleus to the DRt were reported here for the first time.

The ipsilateral projection from the DpMe to the DRt was also reported in a previous study (Jones \& Yang, 1985; see also Veazey \& Severin, 1980) but the contralateral projection, as seen here, was not detected, probably due to the lower sensitivity of the method employed (autoradiography). The bilateral projection from the caudal VLPAG terminating in the DRt was already mentioned (Chen \& Aston-Jones, 1996; Odeh \& Antal, 2001).

\section{Pons}

The projections from the basilar pontine nuclei, SubCV (see Westlund \& Coulter, 1980) and reticulotegmental nucleus of the pons to the DRt were shown here for the first time.

The strong bilateral projection from both the medial and lateral PBN and KF (Saper \& Loewy, 1980; Herbert et al., 1990; Krukoff et al., 1993) and the bilateral projections from the $\mathrm{A}_{5}$ (Tavares et al., 1997) and LC (Jones \& Yang, 1985; Fritschy \& Grzanna, 1990) to the DRt have already been reported.

\section{Cerebellum}

In a previous study, the injection of ${ }^{3} \mathrm{H}$-leucine/proline into the lateral and interposed cerebellar nuclei resulted in fibre and terminal labelling along all the ipsilateral dorsoventral extension of the DRt (Woodson \& Angaut, 1984). Our findings extend these results by demonstrating a bilateral projection to the DRt from all the three groups of deep cerebellar nuclei (medial, interposed and lateral; see also Teune et al., 2000).

\section{Medulla oblongata}

To the best of our knowledge, the projections from the nucleus raphe magnus, inferior olive, paratrigeminal nucleus and ventral reticular nucleus to the DRt are reported here for the first time.

Our study confirms a very strong ipsilateral projection from the caudal part of the spinal trigeminal nucleus to the DRt (Esser et al., 1998), but also shows an important contralateral projection to the nucleus. Contrary to our results, the projection from the $A_{1}$ noradrenergic cell group was previously reported to be bilateral, probably due to the larger injection in the $\mathrm{A}_{1}$ area in that study, which reached the nearby LRt (McKellar \& Loewy, 1982). The latter has a bilateral projection to the DRt, as shown by the present study.

\section{Functional considerations}

The DRt seems to be an important recipient for the nociceptive information transmitted supraspinally as most DRt neurons (Villanueva et al., 1988) and superficial dorsal horn neurons projecting to the DRt (Almeida \& Lima, 1997) are activated by noxious stimulation converging from all the body to the DRt (Villanueva et al., 1988). In addition, the nociceptive input is transmitted to the PF and ventromedial (VM) and reunions thalamic nuclei of the medial thalamus, to which the DRt projects massively (Villanueva et al., 1997; Monconduit et al., 1999). These nuclei project to the amygdala and hippocampus (Ottersen \& Ben-Ari, 1979; Su \& Bentivoglio, 1990), areas involved in emotional/affective and cognitive control (Lopes-da-Silva et al., 1990). They also project to large areas of the motor cortex (Donoghue \& Parham, 1983) and/or dorsal (caudate-putamen complex) and ventral (nucleus accumbens) striatum (Berendse \& Groenewegen, 1990). Altogether, these data indicate that the DRt belongs to the medial pain system (Melzack \& Casey, 1968) and may be involved in processing the motor actions triggered by the motivational-affective afferent component of pain sensation.

The large spectrum of projections converging upon the DRt indicates that the activity of the nucleus is affected by, and involved in, several brain functions. However, in a classification effort, the brain areas projecting to the DRt were considered to belong to one or more of the following two broad functional systems.

Sensorimotor and pain control systems

A large spectrum of brain areas belonging to the supraspinal pain control system project to the DRt and may modulate its activity. Thus, areas shown to be antinociceptive, like the motor cortex, basal ganglia, amygdala, thalamus (PF, paraventricular thalamic nucleus, sensory thalamus), hypothalamus (LH, Pa, Arc, PH), the PAG-RVM circuit, noradrenergic brainstem areas (LC/subcoeruleus, PBN, KF, $\mathrm{A}_{5}, \mathrm{Sol}, \mathrm{A}_{1}$ ) and the brainstem reticular formation ( $\mathrm{DpMe}, \mathrm{CnF}, \mathrm{VRt}$, VLMlat) were shown here to project to the DRt (reviewed by Lima \& Almeida, 2002). On the other hand, areas traditionally known to be antinociceptive but presenting also pronociceptive actions, such as the RVM (Wei et al., 1999; Kovelowski et al., 2000) and Sol (Ren et al., 1990; Ness et al., 2000), also project to the DRt. Moreover, the ipsilateral and contralateral DRt itself, which has a primarily pronociceptive action (Almeida et al., 1996, 1999a), have shortprojecting neurons inside its borders. Altogether, these data indicate that the DRt is a likely candidate to mediate the balance between inhibiting and facilitating nociceptive actions that may, respectively, turn off or turn on the descending facilitating action of the nucleus.

The DRt seems also to be implicated in the pain motor reaction itself, as it not only receives a strong projection from the motor cortex and several extrapyramidal motor areas (globus pallidus, substantia nigra, RD, basilar pontine nuclei, reticulotegmental nucleus of the pons, deep cerebellar nuclei, IO, PCRt and LRt), but also projects to the spinal ventral horn (Villanueva et al., 1995). Thus, it is possible that the DRt influences the motor reactions to noxious stimulation directly by modulating the activity of spinal motoneurons. 


\section{Autonomic and limbic systems}

The activity of DRt neurons can also be influenced by the visceral motor system, which involves afferents from structures such as the dorsal motor nucleus of the vagus and the PrL and IL cortices. Other important forebrain autonomic centres (reviewed by Loewy, 1990) involved in the organism homeostasis and projecting to the DR include the BST, $\mathrm{Ce}, \mathrm{Pa}$ and $\mathrm{LH}$. Most of the above autonomic centres belong also to the limbic system (where emotions are modulated; reviewed by Lopes-da-Silva et al., 1990), namely the prefrontal cortex (PrL, IL and OC), BST, Ce and SI. These data indicate that the DRt not only relays ascending nociceptive information related to the motivational-affective component of pain (see above), but its activity can also be modulated by the reaction triggered by that dimension of pain.

\section{Brain afferents to the DRt: anterograde-tracing studies}

We administered BDA to some of the brain areas that presented numerous retrogradely labelled cells after CTb injections in the DRt; all these injections confirmed, by the presence of abundant fibre- and bouton-labelling, that those areas project in fact to the DRt. Interestingly, the most dorsal portion of the DRt (DRtd; Almeida et al., 1995) is the only, or at least the main, area of the nucleus that receives projections from the various brain areas injected. The DRtd is the area of the nucleus that is reciprocally connected with the spinal cord lamina I through asymmetrical synapses, presumably excitatory (Almeida et al., 1993, 2000) and, thus, may underlie the descending facilitation of spinal nociception conveyed by the nucleus (Almeida et al., 1996, 1999a, 2000; Lima \& Almeida, 2002). Moreover, the DRtd is the area that relays the transmission of ascending nociceptive transmission from the whole body to the VM (Monconduit et al., 1999; Desbois \& Villanueva, 2001) which, in turn, projects diffusely to all areas of the ipsilateral cortex (Herkenham, 1979). Thus, one might conclude that the DRtd is an area of convergence of modulatory actions from the brain upon the ascending nociceptive input carried by the medial pain system.

\section{Conclusion}

In summary, the present findings, together with the current available literature, indicate that the DRtd might be at the centre of: (i) a spinoreticulo-thalamo-cortical nociceptive pathway that spreads the nociceptive information converging from all the body to large areas of the cortex; (ii) a supraspinal network that integrates information from areas of the autonomic, limbic, pyramidal, extrapiramidal and antinociceptive systems before triggering its output action; (iii) a descending nociceptive facilitating pathway that increases nociceptive transmission at the spinal dorsal horn level (Lima \& Almeida, 2002).

\section{Acknowledgements}

This study was supported by BIOTECH project $\mathrm{n}^{\circ}$ BIO4-CT98-007676, the Pain Gulbenkian Programme and Project $n^{\circ}$ POCTI/NSE/38952/2001 from Fundação para a Ciência e Tecnologia (FCT).

\section{Abbreviations}

$3 \mathrm{~V}$, 3rd ventricle; $4 \mathrm{~V}$, 4th ventricle; 7 , facial nucleus; 10 , dorsal motor nucleus of the vagus; 12 , hypoglossal nucleus; A1, A1 noradrenergic cell group; A5, A5 noradrenergic cell group; AI, agranular insular cortex; AIP, agranular insular cortex, posterior part; Aq, aqueduct of Sylvius; Arc, arcuate hypothalamic nucleus; Au1, primary auditory cortex; Au2, secondary auditory cortex; BDA, biotinylated-dextran amine; BL, basolateral amygdaloid nucleus; BPN, basilar pontine nuclei; BST, bed nucleus of the stria terminalis; cc, central canal; $\mathrm{Ce}$, central amygdaloid nucleus; $\mathrm{Cg} 1$, cingulated cortex; $\mathrm{CnF}$, cuneiform nucleus; $\mathrm{CTb}$, cholera toxin subunit $\mathrm{B}$; cu, cuneate fasciculus; $\mathrm{Cu}$, cuneate nucleus; DAB, diaminobenzidine; DI, dysgranular insular cortex; DLPAG, dorsolateral periaqueductal grey; DMPAG, dorsomedial periaqueductal grey; DPGi, dorsal paragigantocellular nucleus; DpMe, deep mesencephalic nucleus; DRt, dorsal reticular nucleus; DRtd, dorsal reticular nucleus, dorsal part; DRV, dorsal raphe nucleus, ventral part; DRVL, dorsal raphe nucleus, ventrolateral part; Ect, ectorhinal cortex; fmi, forceps minor of the corpus callosum; GI, granular insular cortex; Gi, gigantocellular reticular nucleus; GiA, gigantocellular reticular nucleus, alpha part; GiV, gigantocellular reticular nucleus, ventral part; HRP, horseradish peroxidase; ic, internal capsule; IL, infralimbic cortex; Int, interposed cerebellar nucleus; IntA, interposed cerebellar nucleus, anterior part; IntPPC, interposed cerebellar nucleus, posterior parvicellular part; IO, inferior olive; IRt, intermediate reticular nucleus; KF, Kölliker-Fuse nucleus; Lat, lateral (dentate) cerebellar nucleus; LC, locus coeruleus; LGP, lateral globus pallidus; LH, lateral hypothalamic area; LPAG, lateral periaqueductal grey; LPB, lateral parabrachial nucleus; LPGi, lateral paragigantocellular nucleus; LRt, lateral reticular nucleus; M1, primary motor cortex; M2, secondary motor cortex; Me5, mesencephalic trigeminal nucleus; Medical, medial (fastigial) cerebellar nucleus; Mo5, motor trigeminal nucleus; MPB, medial parabrachial nucleus; OC, orbital cortex; Pa5, paratrigeminal nucleus; Pa, paraventricular hypothalamic nucleus; PAG, periaqueductal grey matter; PaMP, paraventricular hypothalamic nucleus, medial parvicellular part; $\mathrm{PaPo}$, paraventricular hypothalamic nucleus, posterior part; $\mathrm{PaR}$, pararubral nucleus; $\mathrm{PB}$, phosphate buffer; PBN, parabrachial nuclei; PBS, phosphate buffer saline; PBST, phosphate buffer saline with triton; PCRt, parvocellular reticular nucleus; PF, parafascicular thalamic nucleus; $\mathrm{PH}$, posterior hypothalamic area; PHA-L, Phaseolus vulgaris-leucoagglutinin; PnC, pontine reticular nucleus, caudal part; PnO, pontine reticular nucleus, oral part; PPTg, pedunculopontine tegmental nucleus; PRh, perirhinal cortex; PrL, prelimbic cortex; Pta, parietal association cortex pyx, pyramidal decussation; RD, red nucleus; RMg, raphe magnus nucleus; RNCD, substantia nigra, compact part; Ro, nucleus of Roller; $\mathrm{ROb}$, raphe obscurus nucleus; RPa, raphe pallidus nucleus; RVM, rostroventromedial medulla; S1, primary somatosensory cortex; S2, secondary somatosensory cortex; scp, superior cerebellar peduncle (brachium conjunctivum); SI, substantia innominata; SNR, substantia nigra, reticular part; sol, solitary tract; Sol, nucleus of the solitary tract; SolM, nucleus of the solitary tract, medial part; SolVL, nucleus tractus solitarius, ventrolateral part; Sp5C, spinal trigeminal nucleus, caudal part; Sp5I, spinal trigeminal nucleus, interpolar part; STh, subthalamic nucleus; SubCV, subcoeruleus nucleus, ventral part; TC, tuber cinereum area; TeA, temporal association cortex; Ve, vestibular nuclei; VLMlat, ventrolateral medulla, lateral part; VLPAG, ventrolateral periaqueductal grey matter; VM, ventromedial thalamic nucleus; VRt, ventral reticular nucleus; WDR, wide-dynamic-range; WGA-ApoHRP$\mathrm{Au}$, wheatgerm agglutinin-apohorseradish peroxidase conjugate; WGA-HRP, wheat germ agglutinin-horseradish peroxidase conjugate; ZI, zona incerta.

\section{References}

Allen, G.V. \& Cechetto, D.F. (1992) Functional and anatomical organization of cardiovascular pressor and depressor sites in the lateral hypothalamic area. I. Descending projections. J. Comp. Neurol., 315, 313-332.

Almeida, A. \& Lima, D. (1997) Activation by cutaneous or visceral noxious stimulation of spinal neurons projecting to the medullary dorsal reticular nucleus in the rat: a c-fos study. Eur. J. Neurosci., 9, 686-695.

Almeida, A., Storkson, R., Lima, D., Hole, K. \& Tjolsen, A. (1999a) The medullary dorsal reticular nucleus facilitates pain behaviour induced by formalin in the rat. Eur. J. Neurosci., 11, 110-122.

Almeida, A., Cobos, A. \& Lima, D. (1999b) Brain afferents to the medullary dorsal reticular nucleus. A CTb retrograde tracing study in the rat [Abstract]. 9th World Congress on Pain (Vienna, 1999), 172.

Almeida, A., Tavares, I. \& Lima, D. (1995) Projection sites of superficial or deep dorsal horn in the dorsal reticular nucleus. Neuroreport, 6, 1245-1248.

Almeida, A., Tavares, I. \& Lima, D. (2000) Reciprocal connections between the medullary dorsal reticular nucleus and the spinal dorsal horn in the rat. Eur. J. Pain, 4, 373-387.

Almeida, A., Tavares, I., Lima, D. \& Coimbra, A. (1993) Descending projections from the medullary dorsal reticular nucleus make synaptic contacts with spinal cord lamina I cells projecting to that nucleus: an electron microscopic tracer study in the rat. Neuroscience, 55, 1093-1106. 
Almeida, A., Tjolsen, A., Lima, D., Coimbra, A. \& Hole, K. (1996) The medullary dorsal reticular nucleus facilitates acute nociception in the rat. Brain Res. Bull., 39, 7-15.

Angelucci, A., Clascá, F. \& Sur, M. (1996) Anterograde axonal tracing with the subunit B of cholera toxin: a highly sensitive immunohistochemical protocol for revealing fine axonal morphology in adult and neonatal brains. J. Neurosci. Meth., 65, 101-112.

Basbaum, A.I. \& Fields, H.L. (1984) Endogenous pain control systems: brainstem spinal pathways and endorphin circuitry. Annu. Rev. Neurosci., 7, 309-338.

Berendse, H.W. \& Groenewegen, H.J. (1990) Organization of the thalamostriatal projections in the rat, with special emphasis on the ventral striatum. J. Comp. Neurol., 299, 187-228.

Bian, D., Ossipov, M.H., Malan,T.P. Jr \& Porreca, F. (1998) Tactile allodynia, but not thermal hyperalgesia, of the hindlimbs is blocked by spinal transection in rats with nerve injury. Neurosci. Lett., 241, 79-82.

Bouhassira, D., Villanueva, L., Bing, Z. \& Le Bars, D. (1992) Involvement of the subnucleus reticularis dorsalis in diffuse noxious inhibitory controls in the rat. Brain Res., 595, 353-357.

Cervero, F., Schaible, H.G. \& Schmidt, R.F. (1991) Tonic descending inhibition of spinal cord neurons driven by joint afferents in normal cats and in cats with inflamed knee-joint. Expl. Brain Res., 83, 675-678.

Chen, S. \& Aston-Jones, G. (1996) Extensive projections from the midbrain periaqueductal gray to the caudal ventrolateral medulla: a retrograde and anterograde tracing study in the rat. Neuroscience, 71, 443-459.

Desbois, C., Le Bars, D. \& Villanueva, L. (1999) Organization of cortical projections to the medullary subnucleus reticularis dorsalis: a retrograde and anterograde tracing study in the rat. J. Comp. Neurol., 410, 178-196.

Desbois, C. \& Villanueva, L. (2001) The organization of lateral ventromedial thalamic connections in the rat: a link for the distribution of nociceptive signals to widespread cortical regions. Neuroscience, 102, 885-898.

Donoghue, J.P. \& Parham, C. (1983) Afferent connections of the lateral agranular field of the rat motor cortex. J. Comp. Neurol., 217, 390-404.

Donovick, P.J. (1974) A metachromatic stain for neural tissue. Stain Technol., 49, 49-51.

Dugast, C., Almeida, A. \& Lima, D. (2000) The medullary dorsal reticular nucleus (DRt) increases the response of lumbar nociceptive neurons to electrical stimulation. Soc. Neurosci. Abstr., 26, 652.

Dugast, C., Almeida, A. \& Lima, D. (2001) Wind-up of spinal cord widedynamic-range neurons is influenced by the medullary dorsal reticular nucleus. Soc. Neurosci. Abstr., 27, 171.1.

Esser, M.J., Pronych, S.P. \& Allen, G.V. (1998) Trigeminal-reticular connections: possible pathways for nociception-induced cardiovascular reflex responses in the rat. J. Comp. Neurol., 391, 526-544.

Fasmer, O.B., Berge, O.G., Tveiten, L. \& Hole, K. (1986) Changes in nociception after 6-hydroxydopamine lesions of the descending catecholaminergic pathways in mice. Pharmacol. Biochem. Behav., 24, 1441-1444.

Fields, H.L. \& Basbaum, A.I. (1999) Central nervous system mechanisms of pain modulation. In Wall, P.D. \& Melzack, C. (eds), Textbook of Pain, 4th edn. Churchill Livingstone, New York, pp. 309-329.

Fields, H.L., Bry, J., Hentall, I. \& Zorman, G. (1983) The activity of neurons in the rostral medulla of the rat during withdrawal from noxious heat. $J$. Neurosci., 3, 2545-2552.

Fritschy, J.-M. \& Grzanna, R. (1990) Distribution of locus coeruleus axons within the rat brainstem demonstrated by Phaseolus vulgaris leucoagglutinin anterograde tracing in combination with dopamine- $\beta$ hydroxylase immunofluorescence. J. Comp. Neurol., 293, 616-631.

Gebhart, G.F. \& Randich, A. (1990) Brainstem modulation of nociception. In Klemm, W.R. \& Vertes, R.P. (eds), Brainstem Mechanisms of Behavior. John Wiley \& Sons, New York, pp. 315-352.

Hammond, D.L. (1986) Control systems for nociceptive afferent processing; the descending inhibitory pathways. In Yaksh, T.L. (ed.), Spinal Afferent Processing. Plenum Press, New York, pp. 363-390.

Herbert, H., Moga, M.M. \& Saper, C.B. (1990) Connections of the parabrachial nucleus with the nucleus of the solitary tract and the medullary reticular formation in the rat. J. Comp. Neurol., 293, 540-580.

Herkenham, M. (1979) The afferent and efferent connections of the ventromedial thalamic nucleus in the rat. J. Comp. Neurol., 183, 487-518.

Jones, S.L. (1992) Descending control of nociception. In Light, A.R. (ed.), The Initial Processing of Pain and its Descending Control.: Spinal and Trigeminal Systems. Karger, New York, pp. 203-295.

Jones, B.E. \& Yang, T.-Z. (1985) The efferent projections from the reticular formation and the locus coeruleus studied by anterograde and retrograde axonal transport in the rat. J. Comp. Neurol., 242, 56-92.
Kauppila, T., Kontinen, V.K. \& Pertovaara, A. (1998) Influence of spinalization on spinal withdrawal reflex responses varies depending on the submodality of the test stimulus and the experimental pathophysiological condition in the rat. Brain Res., 797, 234-242.

Kovelowski, C.J., Ossipov, M.H., Sun, H., Lai, J., Malan,T.P. Jr \& Porreca, F. (2000) Supraspinal cholecystokinin may drive tonic descending facilitation mechanisms to maintain neuropathic pain in the rat. Pain, 87, 265-273.

Krukoff, T.L., Harris, K.H. \& Jhamandas, J.H. (1993) Efferent projections from the parabrachial nucleus demonstrated with the anterograde tracer Phaseolus vulgaris leucoagglutinin. Brain Res. Bull., 30, 163-172.

Lima, D. (1990) A spinomedullary projection terminating in the dorsal reticular nucleus of the rat. Neuroscience, 34, 577-589.

Lima, D. (1998) Anatomical basis for the dynamic processing of nociceptive input. Eur. J. Pain, 2, 195-202.

Lima, D. \& Almeida, A. (2002) The medullary dorsal reticular nucleus as a pronociceptive centre of the pain control system. Prog. Neurobiol., 66, 81108.

Lima, D. \& Coimbra, A. (1988) The spinothalamic system of the rat: structural types of retrogradely labelled neurons in the marginal zone (lamina I). Neuroscience, 27, 215-230.

Lima, D., Mendes-Ribeiro, J.A. \& Coimbra, A. (1991) The spino-lateroreticular system of the rat: projections from the superficial dorsal horn and structural characterization of marginal neurons involved. Neuroscience, $\mathbf{4 5}$, $137-152$.

Loewy, A.D. (1990) Central autonomic pathways. In Loewy, A.D. \& Spyer, K.M. (eds), Central Regulation of Autonomic Functions. Oxford University Press, New York, pp. 88-103.

Lopes-da-Silva, F.H., Witter, M.P., Boeijinga, P.H. \& Lohman, A.M. (1990) Anatomic organization and physiology of the limbic cortex. Physiol. Rev., 70, 453-511.

Luiten, P.G.M., Ter Horst, G.J. \& Steffens, A.B. (1985) The course of paraventricular hypothalamic efferents to autonomic structures in medulla and spinal cord. Brain Res., 329, 374-378.

Luppi, P.H., Fort, P. \& Jouvet, M. (1990) Iontophoretic application of unconjugated cholera toxin $\mathrm{B}$ subunit $(\mathrm{CTb})$ combined with immunocytochemistry of neurochemical substances: a method for transmitter identification of retrogradely labeled neurons. Brain Res., 534, 209-224.

Martin, W.J., Gupta, N.K., Loo, C.M., Rohde, D.S. \& Basbaum, A.I. (1999) Differential effects of neurotoxic destruction of descending noradrenergic pathways on acute and persistent nociceptive processing. Pain, 80, 57-65.

McKellar, S. \& Loewy, A.D. (1982) Efferent projections of the A1 catecholamine cell group in the rat: an autoradiographic study. Brain Res., 241, 11-29.

McNally, G.P. (1999) Pain facilitatory circuits in the mamalian central nervous system: their behavioral significance and role in morphine analgesic tolerance. Neurosci. Biobehav. Rev., 23, 1059-1078.

Melzack, R. \& Casey, K.L. (1968) Sensory, motivational, and central control determinants of pain. A New Conceptual Model. In Kenshalo, D.R. (ed.), The skin senses. Thomas, Springfield, pp. 423-443.

Millan, M.J. (1999) The induction of pain: an integrative review. Prog. Neurobiol., 57, 1-164.

Monconduit, L., Bourgeais, L., Bernard, J.-F., Le Bars, D. \& Villanueva, L. (1999) Ventromedial thalamic neurons convey nociceptive signals from the whole body surface to the dorsolateral neocortex. J. Neurosci., 15, 90639072 .

Ness, T.J., Fillingim, R.B., Randich, A., Backensto, E.M. \& Faught, E. (2000) Low intensity vagal stimulation lowers human thermal pain thresholds. Pain, 86, 81-85.

Odeh, F. \& Antal, M. (2001) The projections of the midbrain periaqueductal grey to the pons and medulla oblongata in rats. Eur. J. Neurosci., 14, 12751286.

Ottersen, O.P. \& Ben-Ari, Y. (1979) Afferent connections to the amygdaloid complex of the rat and cat. J. Comp. Neurol., 187, 401-424.

Paxinos, G. \& Watson, C. (1986) The Rat Brain in Stereotaxic Coordinates, 2nd edn. Academic Press, New York.

Paxinos, G. \& Watson, C. (1998) The Rat Brain in Stereotaxic Coordinates, 4th edn. Academic Press, New York.

Proudfit, H.K. \& Hammond, D.L. (1981) Alterations in nociceptive threshold and morphine-induced analgesia produced by intrathecally administered amine antagonists. Brain Res., 218, 393-399.

Ren, K., Randich, A. \& Gebhart, G.F. (1990) Modulation of spinal nociceptive transmission from nuclei tractus solitarii: a relay for effects of vagal afferent stimulation. J. Neurophysiol., 63, 971-986.

Roy, J.-C., Bing, Z., Villanueva, L. \& Le Bars, D. (1992) Convergence of 
visceral and somatic inputs onto subnucleus reticularis dorsalis neurones in the rat medulla. J. Physiol. (Lond.), 458, 235-246.

Saper, C.B. \& Loewy, A.D. (1980) Efferent connections of the parabrachial nucleus in the rat. Brain Res., 197, 291-317.

Schaible, H.G., Neugebauer, V., Cervero, F. \& Schmidt, R.F. (1991) Changes in tonic descending inhibition of spinal neurons with articular input during the development of acute arthritis in the cat. J. Neurophysiol., 66, 10211032 .

Shepherd (1990) The Synaptic Organization of the Brain. Oxford University Press, London.

Su, H.-S. \& Bentivoglio, M. (1990) Thalamic midline cell populations projecting to the nucleus accumbens, amygdala, and hippocampus in the rat. J. Comp. Neurol., 297, 582-593.

Swanson, L.W. (1998) Brain Maps: Structure of the Rat Brain. Elsevier, New York.

Tavares, I., Lima, D. \& Coimbra, A. (1997) The pontine $A_{5}$ noradrenergic cells which project to the spinal cord dorsal horn are reciprocally connected with the caudal ventrolateral medulla in the rat. Eur. J. Neurosci., 9, 2452 2461.

Teune, T.M., van der Burg, J., van der Moer, J., Voogd, J. \& Ruigrok, T.J. (2000) Topography of cerebellar nuclear projections to the brain stem in the rat. Prog. Brain Res., 124, 141-172.

Tjølsen, A., Berge, O.-G. \& Hole, K. (1991) Lesions of bulbo-spinal serotonergic or noradrenergic pathways reduce nociception as measured by the formalin test. Acta Physiol. Scand., 142, 229-236.

Todd, A.J. \& Spike, R.C. (1993) Localization of classical neurotransmitters and neuropeptides within neurons in laminae I-III of the mammalian spinal dorsal horn. Prog. Neurobiol., 41, 609-645.

Urban, M.O. \& Gebhart, G.F. (1999) Supraspinal contributions to hyperalgesia. Proc. Natl. Acad. Sci., 96, 7687-7692.

Veazey, R.B. \& Severin, C.M. (1980) Efferent projections of the deep mesencephalic nucleus (pars medialis) in the rat. J. Comp. Neurol., 190, 245-258.

Veenman, C.L., Reiner, A. \& Honig, M.G. (1992) Biotinylated dextran amine as an anterograde tracer for single- and double-labeling studies. J. Neurosci. Meth., 41, 239-254.

Villanueva, L., Bernard, J.F. \& Le Bars, D. (1995) Distribution of spinal cord projections from the medullary subnucleus reticularis dorsalis and the adjacent cuneate nucleus: a Phaseolus vulgaris-leucoagglutinin study in the rat. J. Comp. Neurol., 352, 11-32.
Villanueva, L., Bing, Z., Bouhassira, D. \& Le Bars, D. (1989) Encoding of electrical, thermal, and mechanical noxious stimuli by subnucleus reticularis dorsalis neurons in the rat medulla. J. Neurophysiol., 61, 391402.

Villanueva, L., Bouhassira, D., Bing, Z. \& Le Bars, D. (1988) Convergence of heterotopic nociceptive information onto subnucleus reticularis dorsalis neurons in the rat medulla. J. Neurophysiol., 60, 980-1009.

Villanueva, L., Desbois, C., Le Bars, D. \& Bernard, J.-F. (1997) Organization of diencephalic projections from the medullary subnucleus reticularis dorsalis and the adjacent cuneate nucleus: a retrograde and anterograde tracer study in the rat. J. Comp. Neurol., 390, 133-160.

Wei, F., Dubner, R. \& Ren, K. (1999) Nucleus gigantocellularis and nucleus raphe magnus in the brain stem exert opposite effects on behaviora hyperalgesia and spinal fos protein expression after peripheral inflammation. Pain, 80, 127-141.

Westlund, K.N. \& Coulter, J.D. (1980) Descending projections of the locus coeruleus and subcoeruleus/medial parabrachial nuclei in monkey: axonal transport studies and dopamine-beta-hydroxylase immunocytochemistry. Brain Res., 2, 235-264.

Woodson, W. \& Angaut, P. (1984) The distribution and origin of the ipsilateral descending limb of the brachium conjunctivum. An autoradiographic and horseradish peroxidase study in the rat. Expl. Brain Res., 56, 167-182.

Wouterlood, F.G. \& Jorritsma-Byham, B. (1993) The anterograde neuroanatomical tracer biotinylated dextran-amine: comparison with the tracer Phaseolus vulgaris-leucoagglutinin in preparations for electron microscopy. J. Neurosci. Meth., 48, 75-87.

Yaksh, T.L. (1985) Pharmacology of spinal adrenergic systems which modulate spinal nociceptive processing. Pharmacol. Biochem. Behav., 22, 845-858.

Zhuo, M. \& Gebhart, G.F. (1992) Characterization of descending facilitation and inhibition of spinal nociceptive transmission from the nuclei reticularis gigantocellularis and gigantocellularis pars alpha in the rat. $J$. Neurophysiol., 67, 1599-1614.

Zhuo, M. \& Gebhart, G.F. (1997) Biphasic modulation of spinal nociceptive transmission from the medullary raphe nuclei in the rat. J. Neurophysiol., 78, 746-758.

Zimmermann, M. (1983) Ethical guidelines for investigations of experimental pain in conscious animals. Pain, 16, 109-110. 